\title{
A CONTINUOUS VERSION OF THE RELAXATION THEOREM FOR NONLINEAR EVOLUTION INCLUSIONS
}

\author{
By Nikolaos S. PAPAgEORgIOU
}

\begin{abstract}
We consider parametric nonlinear evolution inclusions defined on an evolution triple of spaces. First we prove some continuous dependence results for the solution sets of both the convex and nonconvex problem and for the set of solution-selector parrs of the convex problem. Subsequently, we derive a parametrized version of the Filippov-Gronwall estımate in which the parameter varies in a continuous fashion. Using that estımate, we prove a continuous version of the nonlinear relaxation theorem. An example of a nonlinear parabolic control system is worked out in detail.
\end{abstract}

\section{Introduction}

One of the fundamental results in the theory of differential inclusions (setvalued differential equations), is the "relaxation theorem". It says that if the orientor field (set-valued vector field) is $h$-Lipschitz in the state variable, then the solution set of the differential inclusion is dense in that of the convexified problem (i.e. the system obtained by replacing the original vector field by its closed, convex hull). We refer to the book of Aubin-Cellina [2] (theorem 2, p. 124) which treats differential inclusions in $\boldsymbol{R}^{N}$ and the papers of Papageorgiou [15] and $\mathrm{Zhu}$ [24], which deal with differential inclusions in Banach spaces. Such a density result is important in control theory because it leads to nonlinear versions of the "bang-bang" principle. Recently, the relaxation theorem was extended to evolution inclusions by Frankowska [6] and Papageorgiou [20] for semilinear systems, by Papageorgiou [17] for integrodifferential systems and by Hu-Lakshmikantham-Papageorgiou [9] for nonlinear systems. Furthermore in [16], we studied the relation between relaxability and performance stability for nonlinear variational problems monitored by nonlinear evolution equations. We

1990 AMS Subject classification. Primary 34G20, Secondary 35K55.

Key words and phrases: Evolution triple, compact embedding, continuous selector, Filippov-Gronwall estımate, parabolic system.

Received August 5, 1993 ; revised May 2, 1994. 
should point out that evolution inclusions model partial differential equations with multivalued terms (like distributed parameter and boundary control problems) and so are more appropriate for the analysis of infinite dimensional setvalued systems than the differential inclusions considered by Papageorgiou [15] and $\mathrm{Zhu}$ [24], which do not allow for the presence of unbounded operators (linear or nonlinear) and therefore can not be used in describing systems governed by partial differential equations with multivalued perturbations.

Very recently, Fryszkowski-Rzezuchowski [7] considered differential inclusions in a Banach space, depending on a parameter and established a continuous version of the relaxation theorem. The purpose of this paper is to extend their result to evolution inclusions. In this effort we also prove a continuous dependence result extending Theorem 4.2 of $\mathrm{Zhu}$ [24] and a continuous version of the Filippov-Gronwall inequality, extending this way to a large class of nonlinear systems, an analogous result for semilinear autonomous systems proved by Frankowska [6] and for semilinear nonautonomous systems proved by Papageorgiou $[20]$.

\section{Mathematical preliminaries}

Let $(\Omega, \Sigma)$ be a measurable space and $X$ a separable Banach space. Throughout this work we will be using the following notations:

$$
P_{f(c)}(X)=\{A \subseteq X: \text { nonempty, closed }(\text { convex })\}
$$

and $P_{(w) k(c)}(X)=\{A \subseteq X$ : nonempty, (weakly-) compact, (convex) $\}$.

A multifunction (set-valued function) $F: \Omega \rightarrow P_{f}(X)$ is said to be measurable if for all $x \in X$, the $\boldsymbol{R}_{+}$-valued function $\omega \rightarrow d(x, F(\boldsymbol{\omega}))=\inf \{x-z \|: z \in F(\boldsymbol{\omega})\}$ is measurable. Next let $\mu(\cdot)$ be a finite measure on $(\Omega, \Sigma)$. By $S_{F}^{p}, 1 \leqq p \leqq \infty$, we will denote the set of selectors of $F(\cdot)$ that belong in the Lebesgue-Bochner space $L^{p}(\Omega, X)$; i. e. $S_{F}^{p}=\left\{f \in L^{p}(\Omega, X): f(\boldsymbol{\omega}) \in F(\boldsymbol{\omega}) \mu\right.$-a. e. $\}$. In general, this set may be empty. However it is easy to check using Aumann's selection theorem (see Wagner [22], Theorem 5.10), that if $F: \Omega \rightarrow 2^{X} \backslash\{\emptyset\}$ is a multifunction such that $G r F=\{(\omega, x) \in \Omega \times X: x \in F(\omega)\} \in \Sigma \times B(X)$ (i. e. $F(\cdot)$ is graph measurable), with $B(X)$ being the Borel $\sigma$-field of $X$, then $S_{F}^{p}$ is nonempty if and only if $\omega \rightarrow \inf \{\|z\|: z \in F(\boldsymbol{\omega})\} \in L^{p}(\Omega, \boldsymbol{R})$. Note that for a $P_{f}(X)$-valued multifunction, we have that measurability implies graph measurability, while the converse is true if $\Sigma$ is $\mu$-complete. Also remark that $S_{F^{\prime}}^{p}$ is decomposable in the sense that for all $(A, f, g) \in \Sigma \times S_{F}^{p} \times S_{F}^{p}$ we have $\chi_{A} f+\chi_{A c} g \in S_{F}^{p}$ (here $\chi_{A}(\cdot)$ is the characteristic function of a set $A \in \Sigma$ ).

On $P_{f}(X)$ we can define a generalized metric (i.e., the distance function can take the value $+\infty$; cf. Choquet [25]), known in the literature as the Hausdorff metric, by setting for $A, B \in P_{f}(X)$ 


$$
h(A, B)=\max \left[\sup _{a \in A} d(a, B), \sup _{b \in B} d(b, A)\right]
$$

where $d(a, B)=\inf \{\|a-b\|: b \in B\}$ and $d(b, A)=\inf \{\|b-a\|: a \in A\}$. It is wellknown (see for example Klein-Thompson [10]), that the generalized metric space $\left(P_{f}(X), h\right)$ is complete. A multifunction $F: X \rightarrow P_{f}(X)$ is said to be Hausdorff continuous ( $h$-continuous), if it is continuous from $X$ into the metric space $\left(P_{f}(X), h\right)$.

If $Y, Z$ are Hausdorff topological spaces and $G: Y \rightarrow 2^{Z} \backslash\{0\}$, we say that $G(\cdot)$ is lower semicontinuous (l.s.c.), if for every $C \cong Z$ closed, $G^{+}(C)=$ $\{y \in Y: G(y) \subseteq C\}$ 1s closed (or equivalently for every $U \subseteq Z$ open, $G^{-}(U)=$ $\{y \in Y: G(y) \cap U \neq \emptyset\}$ is open). If $Y, Z$ are metric spaces, then lower semicontinuity is equivalent to saying that if $y_{n} \rightarrow y$ in $Y$, then $G(y) \leqq \underline{\lim } G\left(y_{n}\right)=$ $\left\{z \in Z: \lim d_{Z}\left(z, G\left(y_{n}\right)\right)=0\right\}=\left\{z \in Z: z=\lim z_{n}, z_{n} \in G\left(y_{n}\right), n \geqq 1\right\}$, with $d_{Z}(\cdot, \cdot)$ being the metric on $Z$. Furthermore in this case the lower semicontinuity of $G(\cdot)$ is equivalent to the upper semicontinuity of the distance functions $y \rightarrow$ $d_{Z}(z, G(y))=\inf \left\{d_{Z}\left(z, z^{\prime}\right): z^{\prime} \in G(y)\right\}$ for every $z \in Z$. We say that $G(\cdot)$ is upper semicontinuous (u.s.c.) if for every $C \leqq Z$ closed $G^{-}(C)=\{y \in Y: G(y) \cap C \neq \emptyset\}$ is closed (or equivalently for every $U \subseteq Z$ open $G^{+}(U)=\{y \in Y: G(y) \subseteq U\}$ is open). A multifunction which is both l.s.c. and $u$.s.c. is said to be continuous (or sometimes Vietoris continuous, to emphasize that on the hyperspace $2^{Z} \backslash\{\emptyset\}$ we consider the Vietoris hyperspace topology). If $Y, Z$ are metric spaces and $G(\cdot)$ is $P_{k}(Z)$-valued, then continuity and $h$-contınuity coincide. For further details we refer to DeBlasi-Myjak [5] and Klem-Thompson [10].

Let $H$ be a separable Hilbert space of norm $|\cdot|$. Let $X$ be a reflexive, separable Banach space embedding continuously and densely into $H$. Identifying $H$ with its dual (pivot space), we have that $X \subseteq H \subseteq X^{*}$, with all injections being continuous and dense. Such a triple of spaces is usually known in the literature as "evolution triple" (the names "Gelfand triple" or "spaces in normal position" are also used). We will also assume that the embeddings are compact. In concrete applications, evolution, triples are generated by Sobolev spaces (see the example in section 6). By $\|\cdot\|$ (resp. $\|\cdot\|_{*}$ ) will denote the norm of $X$ (resp. of $\left.X^{*}\right)$. Also by $\langle\cdot, \cdot\rangle$ we will denote the duality brackets for the pair $\left(X, X^{*}\right)$ and by $(\cdot, \cdot)$ the inner product of $H$. The two are compatible in the sense that $(\cdot, \cdot)=\left.\langle\cdot, \cdot\rangle\right|_{X \times H}$. Let $T=[0, b], 1<p, q<\infty, 1 / p+1 / q=1$ and define $W_{p q}(T)=\left\{x \in L^{p}(T, X): x \in \boldsymbol{L}^{q}\left(T, X^{*}\right)\right\}$. The derivative involved in this definition is understood in the sense of vector-valued distributions. Furnished with the norm $\|x\|_{W_{p q}(T)}=\left(\|x\|_{L}^{2} p_{(T, X)}+\|x\|_{L}^{2} q_{(T, X *)}\right)^{1 / 2}, W_{p q}(T)$ becomes a Banach space, which is separable and reflexive. Furthermore $W_{p q}(T)$ embeds continuously into $C(T, H)$ and compactly into $L^{p}(T, H)$. For further details, we refer to Zeidler [23] (Proposition 23.23, pp. 422-423 and p. 450). When $X$ is a Hilbert space too and $p=q=2, W_{p q}(T)=W(T)$ is a separable Hilbert space with inner product $(x, y)_{W(T)}=(x, y)_{L^{2}(T, X)}+(x, y)_{L^{2}\left(T, X^{*}\right)}$.

A map $A: X \rightarrow X^{*}$ is said to be hemicontinuous if and only if for all $x, y, z$ $\in X$, the map $\lambda \rightarrow\langle A(x+\lambda y), z\rangle$ is continuous from $[0,1]$ into $\boldsymbol{R}$ (i. e. $x \rightarrow A(x)$ 
is continuous from $X$ into $X_{w}^{*}$ along rays; here $X_{w}^{*}$ denotes the space $X^{*}$ furnished with the weak topology). If $A(\cdot)$ is demicontinuous (i.e. when $x_{n} \rightarrow x$ in $X$, we have $A\left(x_{n}\right) \stackrel{w}{\rightarrow} A(x)$ in $X^{*}$ ), then $A(\cdot)$ is hemicontinuous (see Zeidler [23], p. 596). We say that $A(\cdot)$ is monotone, if for all $x, y \in X,\langle A(x)-A(y)$, $x-y\rangle \geqq 0$.

Next let $T=[0, b], \Lambda$ a complete metric space (the parameter space) and $\left(X, H, X^{*}\right)$ an evolution triple of spaces with compact embeddings. The multivalued Cauchy problem under consideration is the following:

$$
\left\{\begin{aligned}
\dot{x}(t)+A(t, x(t)) & \in F(t, x(t), \lambda) a . e . \\
x(0) & =v(\lambda),
\end{aligned}\right\}
$$

where $v: A \rightarrow H$ is given.

In conjunction with (1) we also consider its convexified counterpart:

$$
\left\{\begin{array}{c}
\dot{x}(t)+A(t, x(t)) \in \overline{\operatorname{conv}} F(t, x(t), \lambda) a . e . \\
x(0)=v(\lambda) .
\end{array}\right\}
$$

Here $\overline{c o n v} F(t, x, \lambda)$ denotes the closed, convex hull of the set $F(t, x, \lambda)$. By a solution of (1) (resp. of (2)), we mean a function $x(\cdot) \in W_{p q}(T)$ such that $\dot{x}(t)+A(t, x(t))=f(t)$ a.e., $x(0)=v(\lambda)$, with $f \in L^{q}(T, H), f(t) \in F(t, x(t), \lambda)$ a.e. (resp. $f(t) \in \overline{\operatorname{conv}} F(t, x(t), \lambda)$ a.e.). By $S(\lambda) \subseteq W_{p q}(T)$ we will denote the solution set of $(1)$ and by $S_{r}(\lambda) \cong W_{p q}(T)$ we will denote the solution set of (2).

The following hypothesis on the operator $A(t, x)$ will be useful throughout this work:

$H(A): \quad A: T \times X \rightarrow X^{*}$ is an operator such that

(1) $t \rightarrow A(t, x)$ is measurable,

(2) $x \rightarrow A(t, x)$ is hemicontinuous, monotone,

(3) $\|A(t, x)\|_{*} \leqq a_{1}(t)+c_{1}\|x\|^{p-1} a . e$. with $a_{1}(\cdot) \in L^{p}(T, \boldsymbol{R}), c_{1}>0,2 \leqq p<\infty$, $1 / p+1 / q=1$,

(4) $c\|x\|^{p} \leqq\langle A(t, x), x\rangle$ for all $x \in X$ and almost all $t \in T$ and with $c>0$.

Let $h \in L^{q}(T, H)$. By $w(h, \lambda)(\cdot) \in W_{p q}(T) \subseteq C(T, H)$ we will denote the unique solution of the Cauchy problem $x(t)+A(t, x(t))=h(t)$ a.e., $x(0)=v(\lambda)$. Assuming hypothesis $H(A)$ above, the existence and uniqueness of $w(h, \lambda)(\cdot)$ follows from Theorem 30.A, p. 771 of Zeidler [23].

Then let

and

$$
P(\lambda)=\left\{[x, h] \in W_{p q}(T) \times L^{q}(T, H): x=w(h, \lambda), h \in S_{F^{\prime}(\cdot, x(\bullet), \lambda)}^{q}\right\}
$$

$$
P_{r}(\lambda)=\left\{[x, h] \in W_{p q}(T) \times L^{q}(T, H): x=w(h, \lambda), h \in S_{c \overline{n v} F(\cdot, x(\cdot), 1)}^{q}\right\}
$$

i. e. the sets of solution-selection pairs for Cauchy problems (1) and (2) respectively. 


\section{Continuous dependence results}

In this section we establish the continuity properties of the multifunctions $\lambda \rightarrow P_{r}(\lambda), \lambda \rightarrow S_{r}(\lambda)$ and $\lambda \rightarrow S(\lambda)$. To this end, we will need the following hypothesis on the parametrized orientor field $F(t, x, \lambda)$ :

$H(F)_{1}: \quad F: T \times H \times \Lambda \rightarrow P_{f}(H)$ is a multifunction such that

(1) $t \rightarrow F(t, x, \lambda)$ is measurable,

(2) $h(F(t, x, \lambda), F(t, y, \lambda)) \leqq k_{B}(t)|x-y|$ a.e. for all $\lambda \in B \leqq \Lambda$ compact and with $k_{B} \in L^{q}(T, \boldsymbol{R})$,

(3) $|F(t, x, \lambda)|=\sup \{|v|: v \in F(t, x, \lambda)\} \leqq a_{B}(t)+c_{B}(t)|x|^{2 / q}$ a.e. for all $\lambda \in B \subseteq \Lambda$ compact with $a_{B}, c_{B} \in L^{q}(T, \boldsymbol{R})$,

(4) $\lambda \rightarrow \overline{\operatorname{conv}} F(t, x, \lambda)$ is $l$.s. $c$.

Also we will make the following hypothesis concerning the initial datum $v(\lambda)$ :

$H_{0}: \lambda \rightarrow v(\lambda)$ is continuous from $\Lambda$ into $H$.

From Hu-Lakshmikantham-Papageorgiou [9], we know that for all $\lambda \in A$, $S_{r}(\lambda) \in P_{k}\left(L^{p}(T, H)\right)$ and $S_{r}(\lambda)=\overline{S(\lambda)}$, the closure taken in $L^{p}(T, H)$ (in fact if $X$ is a Hilbert space too and $p=q=2$, then $S_{r}(\lambda) \in P_{k}(C(T, H))$ and $S_{r}(\lambda)=\overline{S(\lambda)}$, the closure taken in $C(T, H)$; this follows from the compact embedding of $W_{2 \infty}(T)$ into $C(T, H)$, see Papageorgiou [11] and Simon [21]). Furthermore given $\lambda \in A$, $P_{r}(\lambda)$ is a nonempty, compact subset of $L^{p}(T, H) \times L^{q}(T, H)_{w}$, where $L^{q}(T, H)_{w}$ denotes the Lebesgue-Bochner space $L^{q}(T, H)$ equipped with the weak topology. Also it is easy to check that for all $\lambda \in A, P_{r}(\lambda) \in P_{f}\left(L^{p}(T, H) \times L^{q}(T, H)\right)$.

THEOREM 3.1. If hypotheses $H(A), H(F)_{1}$ and $H_{0}$ hold,

$$
\text { then } \lambda \rightarrow P_{r}(\lambda) \text { is l.s.c. from } \Lambda \text { into } P_{f}\left(C(T, H) \times L^{q}(T, H)\right) \text {. }
$$

Proof. We need to show that if $\lambda_{n} \rightarrow \lambda$, then $P_{r}(\lambda) \leqq \varliminf P_{r}\left(\lambda_{n}\right)$ (see section $2)$. To this end, let $[x, f] \in P_{r}(\lambda)$. Then by definition we have

$$
\left\{\begin{array}{c}
\dot{x}(t)+A(t, x(t))=f(t) \text { a.e. } \\
x(0)=v(\lambda)
\end{array}\right\}
$$

with $f \in L^{q}(T, H), f(t) \in \overline{\operatorname{conv}} F(t, x(t), \lambda)$ a.e.

Now let $m\left(t, \lambda_{n}\right)=\operatorname{proj}\left(f(t) ; \overline{c o n v} F\left(t, x(t), \lambda_{n}\right)\right)$ and $u\left(t, z, \lambda_{n}\right)=\operatorname{proj}\left(m\left(t, \lambda_{n}\right)\right.$; $\left.\overline{\operatorname{conv}} F\left(t, z, \lambda_{n}\right)\right)$, where $\operatorname{proj}(\cdot ; \overline{\operatorname{conv}} F(t, x, \lambda))$ denotes the metric projection on the closed convex set $\overline{\operatorname{conv}} F(t, x, \lambda) \subseteq H,(t, x, \lambda) \in T \times H \times \Lambda$. From Lemma $\alpha$ of Papageorgiou [18], we know that $t \rightarrow m\left(t, \lambda_{n}\right), u\left(t, z, \lambda_{n}\right) n \geqq 1$ are measurable, while $z \rightarrow u\left(t, z, \lambda_{n}\right) n \geqq 1$ is continuous.

Let $x_{n} \in W_{p q}(T)$ be a solution of the Cauchy problem 


$$
\left\{\begin{array}{c}
\dot{x}_{n}(t)+A\left(t, x_{n}(t)\right)=u\left(t, x_{n}(t), \lambda_{n}\right) \text { a.e. } \\
x_{n}(0)=v\left(\lambda_{n}\right)
\end{array}\right\}
$$

Its existence follows from Theorem 3.1 of Papageorgiou [19], (see also Theorem 3.1 of Hu-Lakshmikantham-Papageorgiou [9]). Clearly $x_{n}(\cdot) \in S\left(\lambda_{n}\right)$. As before exploiting the monotoncity of the operator $A(t, \cdot)$, we have

$$
\begin{gathered}
\frac{1}{2} \frac{d}{d t}\left|x_{n}(t)-x(t)\right|^{2} \leqq\left(u\left(t, x_{n}(t), \lambda_{n}\right)-f(t), x_{n}(t)-x(t)\right) \text { a.e. } \\
\Rightarrow \frac{1}{2}\left|x_{n}(t)-x(t)\right|^{2} \leqq \frac{1}{2}\left|v\left(\lambda_{n}\right)-v(\lambda)\right|^{2}+\int_{0}^{t}\left|u\left(s, x_{n}(s), \lambda_{n}\right)-f(s)\right| \cdot\left|x_{n}(s)-x(s)\right| d s \\
\Rightarrow\left|x_{n}(t)-x(t)\right| \leqq\left|v\left(\lambda_{n}\right)-v(\lambda)\right|+\int_{0}^{t}\left|u\left(s, x_{n}(s), \lambda_{n}\right)-f(s)\right| d s
\end{gathered}
$$

Observe that

(see Brezis [4], Lemma A.5, p. 157).

$$
\begin{aligned}
& \int_{0}^{t}\left|u\left(s, x_{n}(s), \lambda_{n}\right)-f(s)\right| d s \\
& \quad \leqq \int_{0}^{t}\left|u\left(s, x_{n}(s), \lambda_{n}\right)-m\left(s, \lambda_{n}\right)\right| d s+\int_{0}^{t}\left|m\left(s, \lambda_{n}\right)-f(s)\right| d s \\
& \quad \leqq \int_{0}^{t} h\left(F\left(s, x_{n}(s), \lambda_{n}\right), F\left(s, x(s), \lambda_{n}\right)\right) d s+\int_{0}^{t}\left|m\left(s, \lambda_{n}\right)-f(s)\right| d s \\
& \quad \leqq \int_{0}^{t} k_{B}(s)\left|x_{n}(s)-x(s)\right| d s+\int_{0}^{t} d\left(f(s), \overline{\operatorname{conv}} F\left(s, x(s), \lambda_{n}\right)\right) d s
\end{aligned}
$$

with $B=\left\{\lambda_{n}, \lambda\right\}_{n \geqq 1} \cong \Lambda$ compact.

Since by hypothesis $H(F)_{1}(4), \lambda \rightarrow \overline{\operatorname{conv}} F(s, x, \lambda)$ is $l . s . c . \Rightarrow \lambda \rightarrow d(f(s)$, $\overline{\operatorname{conv}} F(s, x(s), \lambda)$ ) is $u$.s. c. (see section 2). Hence because $f(s) \in \overline{\operatorname{conv}} F(s, x(s), \lambda)$ a.e., via Fatou's lemma, we get that

$$
\int_{0}^{t} d\left(f(s), \overline{\operatorname{conv}} F\left(s, x(s), \lambda_{n}\right)\right) d s \rightarrow 0 \quad \text { as } n \rightarrow \infty,
$$

Thus, given $\varepsilon>0$, we can find $n_{0}(\varepsilon) \geqq 1$ such that for $n \geqq n_{0}(\varepsilon)$, we have

$$
\begin{gathered}
\left|x_{n}(t)-x(t)\right| \leqq \varepsilon+\int_{0}^{t} k_{B}(s)\left|x_{n}(s)-x(s)\right| d s \\
\Rightarrow\left\|x_{n}-x\right\|_{C(T, H)} \leqq \varepsilon\left\|k_{B}\right\|_{1} \quad \text { (by Gronwall's inequality) } \\
\Rightarrow x_{n} \rightarrow x \quad \text { as } n \rightarrow \infty \text { in } C(T, H) .
\end{gathered}
$$

Next let $g_{n}(\cdot)=u\left(\cdot, x_{n}(\cdot), \lambda_{n}\right) \in L^{q}(T, H), n \geqq 1$. Then $\left[x_{n}, g_{n}\right] \in P_{r}\left(\lambda_{n}\right), n \geqq 1$. Also we have 


$$
\begin{aligned}
& \int_{0}^{b}\left|u\left(t, x_{n}(t), \lambda_{n}\right)-f(t)\right|^{q} d t \\
& \quad \leqq 2^{q} \int_{0}^{b}\left|u\left(t, x_{n}(t), \lambda_{n}\right)-m\left(t, \lambda_{n}\right)\right|^{q} d t+2^{q} \int_{0}^{b}\left|m\left(t, \lambda_{n}\right)-f(t)\right|^{q} d t \\
& \quad \leqq 2^{q} \int_{0}^{b} h\left(F\left(t, x_{n}(t), \lambda_{n}\right), F\left(t, x(t), \lambda_{n}\right)\right)^{q} d t+2^{q} \int_{0}^{b} d\left(f(t), \overline{\operatorname{conv}} F\left(t, x(t), \lambda_{n}\right)\right)^{q} d t \\
& \quad \leqq 2^{q} \int_{0}^{b} k_{B}(t)^{q}\left|x_{n}(t)-x(t)\right|^{q} d t+2^{q} \int_{0}^{b} d\left(f(t), \overline{\operatorname{conv}} F\left(t, x(t), \lambda_{n}\right)\right)^{q} d t \rightarrow 0 \text { as } n \rightarrow \infty .
\end{aligned}
$$

So $\left[x_{n}, g_{n}\right] \rightarrow[x, f]$ in $C(T, H) \times L^{q}(T, H)$ and $\left[x_{n}, g_{n}\right] \in P_{r}\left(\lambda_{n}\right)$ for all $n \geqq 1$. Therefore we have proved that $P_{r}(\lambda) \leqq \varliminf P_{r}\left(\lambda_{n}\right) \Rightarrow \lambda \rightarrow P_{r}(\lambda)$ 1s $l$.s.c. from $\Lambda$ into $P_{f}\left(C(T, H) \times L^{q}(T, H)\right)$.

Q. E. D.

From the above proof we also get the following result concerning multifunction $\lambda \rightarrow S_{r}(\lambda)$.

THEOREM 3.2. If hypotheses $H(A), H(F)_{1}$ and $H_{0}$ hold,

$$
\text { then } \lambda \rightarrow S_{r}(\lambda) \text { is l.s. c. from } \Lambda \text { into } P_{f}(C(T, H)) \text {. }
$$

In addition, recalling that $S_{r}(\lambda)=\overline{S(\lambda)}$, the closure taken in $C(T, H)$ (see HuLakshmikantham-Papageorgiou [9]) and using Proposition 7.3.3, p. 85 of KleinThompson [10], we also get:

THEOREM 3.3. If hypotheses $H(A), H(F)_{1}$ and $H_{0}$ hold, then $\lambda \rightarrow S(\lambda)$ is l.s. c. from $\Lambda$ into $2^{C(T, H)} \backslash\{\emptyset\}$.

\section{A continuous Filippov-Gronwall estimate}

In this section we prove a parametric version of the Filippov-Gronwall inequality, with the dependence on the parameter being continuous. Recall that the Filippov-Gronwall inequality produces estimates that are useful in the study of the qualitative properties of differential inclusions (see Aubin-Cellina [2], Theorem 1, pp. 120-121). For evolution inclusions, the only such results were proved by Frankowska [6] (Theorem 1.2) and Papageorgiou [20] (Theorem 4.1), for semi-linear systems with no parameter $\lambda \in \Lambda$ present.

We will need the following stronger hypothesis on the orientor field $F(t, x)$ :

$H(F)_{2}: \quad F: T \times H \times \Lambda \rightarrow P_{f}(H)$ is a multifunction such that

(1) $t \rightarrow F(t, x, \lambda)$ is measurable,

(2) $h(F(t, x, \lambda), F(t, y, \lambda)) \leqq k(t)|x-y|$ a.e. for all $\lambda \in \Lambda$ and with $k \in$ $L_{+}^{1}(T, R)$, 
(3) $|F(t, x, \lambda)|=\sup \{|v|: v \in F(t, x, \lambda)\} \leqq a(t)+c(t)|x|^{2 / q}$ a.e. with $a, c$ $\in L^{q}(T, \boldsymbol{R})$

(4) $x \rightarrow F(t, x, \lambda)$ is l.s.c.

Suppose $\lambda \rightarrow[y(\lambda), g(\lambda)]$ is a continuous map from $\Lambda$ into $C(T, H) \times L^{q}(T, H)$. Then we can find $p_{g}: \Lambda \rightarrow L^{q}(T, \boldsymbol{R})$ a continuous map such that

$$
d(g(\lambda)(t), F(t, y(\lambda)(t), \lambda)) \leqq p_{g}(\lambda)(t) \text { a.e. }
$$

We can take, for example, $p_{g}(\lambda)(t)=|g(\lambda)(t)|+a(t)+c(t)|y(\lambda)(t)|^{2 / q}$.

THEOREM 4.1. If hypotheses $H(A), H(F)_{2}$ and $H_{0}$ hold, $\lambda \rightarrow[y(\lambda), g(\lambda)]$ is a continuous map from $\Lambda$ into $C(T, H) \times L^{q}(T, H)$ with $y(\lambda)$ $=w(g(\lambda), \lambda), \varepsilon>0$ and $p_{g}: \Lambda \rightarrow L^{q}(T, \boldsymbol{R})$ is a continuous map satısfying (3) above,

then there exists $\lambda \rightarrow[x(\lambda), r(\lambda)]$ a continuous map from $\Lambda$ into $C(T, H) \times L^{q}(T, H)$ such that for all $\lambda \in \Lambda,[x(\lambda), r(\lambda)] \in$ $P(\lambda)$ and

$$
\begin{gathered}
|x(\lambda)(t)-y(\lambda)(t)| \leqq b \varepsilon e^{\theta(t)}+\int_{0}^{t} p_{g}(\lambda)(s) \exp (\theta(t)-\theta(s)) d s, \quad t \in T \\
\text { with } \theta(t)=\int_{0}^{t} k(s) d s .
\end{gathered}
$$

Proof. Let $E_{0}(\lambda)(t)=\left\{v \in F(t, y(\lambda)(t), \lambda):|v-g(\lambda)(t)|<p_{g}(\lambda)(t)+\varepsilon\right\} . \quad$ Clearly $E_{0}(\lambda)(t) \neq \emptyset$ for almost all $t \in T$ and by redefining $E_{0}(\lambda)(\cdot)$ on a Lebesgue null subset of $T$, we may assume without any loss of generality that $E_{0}(\lambda)(t) \neq 0$ for all $t \in T$. From hypotheses $H(F)_{2}(1)$ and (2) and Theorem 3.3 of Papageorgiou [13], we know that $(t, x) \rightarrow F(t, x, \lambda)$ is measurable $\Rightarrow t \rightarrow F(t, y(\lambda)(t), \lambda)$ is measurable $\Rightarrow \operatorname{Gr} E_{0}(\lambda)(\cdot)=\left\{(t, v) \in G r F(\cdot, y(\lambda)(\cdot), \lambda):|v-g(\lambda)(t)|<p_{g}(\lambda)(t)+\varepsilon\right\} \in \mathcal{L}(T)$ $\times B(H)$ with $\mathcal{L}(T)$ being the Lebesgue $\sigma$-field of $T$ (i.e. the completion with respect to the Lebesgue measure on $T$, of the Borel $\sigma$-field $B(T)$ ). So we can apply Aumann's selection theorem (see Wagner [22], Theorem 5.10), to get $z: T \rightarrow H$ a measurable map such that $z(t) \in E_{0}(\lambda)(t)$ for all $t \in T$. Then define $K_{0}: \Lambda \rightarrow 2^{L^{1}(T, H)}$ by

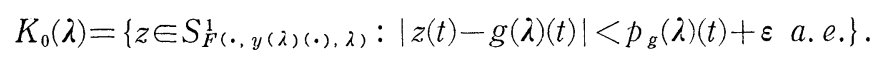

From what was said above, we see that $K_{0}(\lambda) \neq 0$ for all $\lambda \in \Lambda$. Also from Proposition 4 of Bressan-Colombo [3], we know that $\lambda \rightarrow K_{0}(\lambda)$ is l.s.c. and has decomposable values. So $\lambda \rightarrow \overline{K_{0}(\lambda)}$ is l.s.c. with decomposable values from $A$ into $L^{1}(T, H)$. Apply Theorem 3 of Bressan-Colombo [3], to get $r_{0}: \Lambda \rightarrow$ $L^{1}(T, H)$ a continuous map such that $r_{0}(\lambda) \in \overline{K_{0}(\lambda)}$ for all $\lambda \in A$. So $\mid r_{0}(\lambda)(t)$ $g(\lambda)(t) \mid \leqq p_{g}(\lambda)(t)+\varepsilon$ a.e. for all $\lambda \in \Lambda$. Let $x_{1}(\lambda)(\cdot) \in W_{p q}(T) \leqq C(T, H)$ be the unique solution of the Cauchy problem 


$$
\left\{\begin{array}{c}
\dot{x}(t)+A(t, x(t))=r_{0}(\lambda)(t) a . e . \\
x(0)=v(\lambda)
\end{array}\right\}
$$

i. e. $x_{1}(\lambda)=w\left(r_{0}(\lambda), \lambda\right)$. Our claim is that inductively, we can produce two sequences $\left\{x_{n}(\lambda)\right\}_{n \geqq 1} \subseteq W_{p q}(T)$ and $\left\{r_{n-1}(\lambda)\right\}_{n \geqq 1} \subseteq L^{q}(T, H)$ such that

(i) $x_{n}(\lambda)=w\left(r_{n-1}(\lambda), \lambda\right)$,

(ii) $\lambda \rightarrow x_{n}(\lambda)$ is continuous from $\Lambda$ into $C(T, H)$ and $\lambda \rightarrow r_{n-1}(\lambda)$ is continuous from $\Lambda$ into $L^{q}(T, H)$,

(iii) $r_{n-1}(\lambda)(t) \in F\left(t, x_{n-1}(\lambda)(t), \lambda\right)$ a.e.,

(iv) $\left|r_{n-2}(\lambda)(t)-r_{n-1}(\lambda)(t)\right| \leqq k(t) \gamma_{n-1}(\lambda)(t)$ a.e. with

$$
\gamma_{n-1}(\lambda)(t)=\int_{0}^{t} p_{g}(\lambda)(s) \frac{(\theta(t)-\theta(s))^{n-2}}{(n-2) !} d s+b\left(\sum_{k=0}^{n-1} \frac{\varepsilon}{2^{k+1}}\right) \frac{\theta(t)^{n-2}}{(n-2) !} .
$$

From the first part of the proof we know that this is true for $n=1$ (declare $r_{-1}(\lambda)(\cdot)$ to be equal to $g(\lambda)(\cdot)$ and $\gamma_{0}(\lambda)(\cdot)$ equal to $p_{g}(\lambda)(\cdot)+\varepsilon$; without loss of generality, we can assume that $k(t) \geqq 1$ a.e.). Suppose we have obtained $\left\{x_{k}(\lambda)\right\}_{k=1}^{n} \subseteq W_{p q}(T)$ and $\left\{r_{k-1}(\lambda)\right\}_{k=1}^{n} \subseteq L^{q}(T, H)$ satisfy (i) $\rightarrow$ (iv) with $n>1$ (induc-

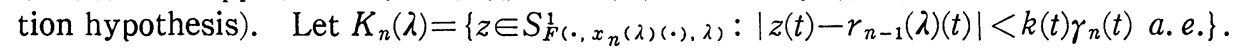
First we will show that for all $\lambda \in \Lambda, K_{n}(\lambda) \neq \emptyset$. Indeed, as before, via Aumann's

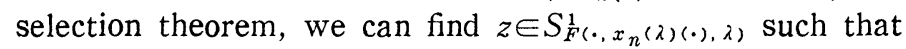

$$
\begin{gathered}
\left|z(t)-r_{n-1}(\lambda)(t)\right|=d\left(r_{n-1}(\lambda)(t), F\left(t, x_{n}(\lambda)(t), \lambda\right)\right) \\
\leqq h\left(F\left(t, x_{n}(\lambda)(t), \lambda\right), F\left(t, x_{n-1}(\lambda)(t), \lambda\right)\right) \\
\leqq k(t)\left|x_{n}(\lambda)(t)-x_{n-1}(\lambda)(t)\right| \text { a.e. }
\end{gathered}
$$

Also from the monotonicity of the operator $A(t, \cdot)$, we have

$$
\begin{gathered}
\left|x_{n}(\lambda)(t)-x_{n-1}(\lambda)(t)\right| \leqq \int_{0}^{t}\left|r_{n-1}(\lambda)(s)-r_{n-2}(\lambda)(s)\right| d s \\
\leqq \int_{0}^{t} k(s) \gamma_{n-1}(\lambda)(s) d s \text { (by the induction hypothesis) } \\
\leqq \int_{0}^{t} k(s) \int_{0}^{s} p_{g}(\lambda)(\tau) \frac{(\theta(s)-\theta(\tau))^{n-2}}{(n-2) !} d \tau d s+b\left(\sum_{k=0}^{n-1} \frac{\varepsilon}{2^{k+1}}\right) \int_{0}^{t} k(s) \frac{\theta(s)^{n-2}}{(n-2) !} d s \\
=\int_{0}^{t} p_{g}(\lambda)(s) \int_{s}^{t} k(\tau) \frac{(\theta(\tau)-\theta(s))^{n-2}}{(n-2) !} d \tau d s+b\left(\sum_{k=0}^{n-1} \frac{\varepsilon}{2^{k+1}}\right) \frac{\theta(t)^{n-1}}{(n-1) !} \\
=\int_{0}^{t} p_{g}(\lambda)(s) \frac{(\theta(t)-\theta(s))^{n-1}}{(n-1) !} d s+b\left(\sum_{k=0}^{n-1} \frac{\varepsilon}{2^{k+1}}\right) \frac{\theta(t)^{n-1}}{(n-1) !}<\gamma_{n}(\lambda)(t) \text { a.e. }
\end{gathered}
$$

So we have

$$
\left|z(t)-\gamma_{n-1}(\lambda)(t)\right|<k(t) \gamma_{n}(\lambda)(t) \text { a.e. }
$$

Hence for every $\lambda \in \Lambda, K_{n}(\lambda) \neq \emptyset$ and as before $\lambda \rightarrow K_{n}(\lambda)$ is $l$. s.c. with decom- 
posable values $\Rightarrow \lambda \rightarrow \overline{K_{n}(\lambda)}$ is $l$.s. c. with decomposable values. Invoking Theorem 3 of Bressan-Colombo [3], we get $r_{n}: \Lambda \rightarrow L^{1}(T, H)$ a continuous map such that $r_{n}(\lambda) \in \overline{K_{n}(\lambda)}$ for all $\lambda \in \Lambda$. So $\left\|r_{n}(\lambda)(t)-r_{n-1}(\lambda)(t)\right\| \leqq k(t) r_{n}(t)$ a.e., $r_{n}(\lambda)(t) \in$ $F\left(t, x_{n}(\lambda)(t), \lambda\right)$ a.e. Set $x_{n+1}(\lambda)=w\left(r_{n}(\lambda), \lambda\right)$. Since $\lambda \rightarrow r_{n}(\lambda)$ is continuous from $\Lambda$ into $L^{1}(T, H)$ (hence into $L^{q}(T, H)$ too, because of hypothesis $H(F)_{2}(3)$ ) and because $w(\cdot, \cdot)$ is continuous from $L^{q}(T, H) \times \Lambda$ into $C(T, H)$, we get that $\lambda \rightarrow x_{n+1}(\lambda)$ is continuous from $\Lambda$ into $C(T, H)$. So by induction we have established the existence of two sequences $\left\{x_{n}(\lambda)\right\}_{n \geqq 1} \leqq W_{p q}(T)$ and $\left\{r_{n-1}(\lambda)\right\}_{n \geqq 1} \subseteq$ $L^{q}(T, H)$ satisfying (i)-(iv) above. Then we have

$$
\begin{gathered}
\int_{0}^{b}\left|r_{n}(\lambda)(t)-r_{n-1}(\lambda)(t)\right| d t \\
\leqq \int_{0}^{b} k(t) \gamma_{n}(\lambda)(t) d t \\
\leqq \int_{0}^{b} k(t) \int_{0}^{t} p_{g}(\lambda)(s) \frac{(\theta(t)-\theta(s))^{n-1}}{(n-1) !} d s d t+b\left(\sum_{k=0}^{n} \frac{\varepsilon}{2^{k+1}}\right) \int_{0}^{b} k(t) \frac{\theta(t)^{n-1}}{(n-1) !} d t \\
\leqq \frac{\theta(b)^{n-1}}{(n-1) !}\left\|p_{g}(\lambda)\right\|_{1} \cdot\|k\|_{1}+b^{2}\left(\sum_{k=0}^{n} \frac{\varepsilon}{2^{k+1}}\right) \frac{\theta(b)^{n-1}}{(n-1) !}\|k\|_{1} .
\end{gathered}
$$

Also for all $(\lambda, t) \in \Lambda \times T$, we have

$$
\left|x_{n+1}(\lambda)(t)-x_{n}(\lambda)(t)\right| \leqq\left\|r_{n}(\lambda)-r_{n-1}(\lambda)\right\|_{L^{1}(T, H)} .
$$

Hence we deduce that $\left\{x_{n}(\lambda)\right\}_{n \geqq 1} \subseteq C(T, H)$ and $\left\{r_{n-1}(\lambda)\right\}_{n \geq 1} \subseteq L^{1}(T, H)$ are both Cauchy sequences. Furthermore, recall that $\lambda \rightarrow p_{g}(\lambda)$ is continuous from $\Lambda$ into $L^{q}(T, H)$, hence locally bounded, and so the above Cauchy property is uniform for $\lambda \in B \subseteq \Lambda$ compact. So we get

$$
\begin{gathered}
x_{n}(\lambda) \rightarrow x(\lambda) \quad \text { in } C(T, H) \\
\text { and } \quad r_{n}(\lambda) \rightarrow r(\lambda) \quad \text { in } L^{1}(T, H) \text { as } n \rightarrow \infty,
\end{gathered}
$$

and both $\lambda \rightarrow x(\lambda)$ and $\lambda \rightarrow r(\lambda)$ are continuous. In addition, note that because of hypothesis $H(F)_{2}(2) r(\lambda)(t) \in F(t, x(\lambda)(t), \lambda)$ a.e. and so by hypothesis $H(F)_{2}(3)$

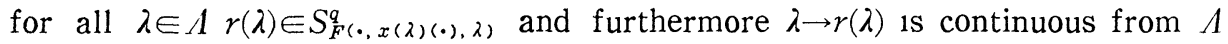
into $L^{q}(T, H)$. In addition, if $z(\lambda)=w(r(\lambda), \lambda)$, then as before, thanks to the monotonicity of $A(t, \cdot)$, we have

$$
\begin{aligned}
\left|x_{n}(\lambda)(t)-z(\lambda)(t)\right| & \leqq \int_{0}^{b}\left|r_{n-1}(\lambda)(s)-r(\lambda)(s)\right| d s \rightarrow 0 \quad \text { as } n \rightarrow \infty \\
& \Rightarrow x_{n}(\lambda) \rightarrow z(\lambda) \quad \text { in } C(T, H) \\
& \Rightarrow z(\lambda)=x(\lambda) \quad \text { for all } \lambda \in \Lambda .
\end{aligned}
$$

So we have established that for all $\lambda \in \Lambda,[x(\lambda), r(\lambda)] \in P(\lambda)$.

Next from the triangle inequality, we have 


$$
\left|y(\lambda)(t)-x_{n}(\lambda)(t)\right| \leqq\left|y(\lambda)(t)-x_{1}(\lambda)(t)\right|+\sum_{k=1}^{n-1}\left|x_{k}(\lambda)(t)-x_{k+1}(\lambda)(t)\right| .
$$

Recall that

$$
\begin{gathered}
\left|x_{k}(\lambda)(t)-x_{k+1}(\lambda)(t)\right| \leqq \int_{0}^{t}\left|r_{k-1}(\lambda)(s)-r_{k}(\lambda)(s)\right| d s \\
\leqq \int_{0}^{t} k(s) \gamma_{k}(s) d s \\
\leqq \int_{0}^{t} k(s) \int_{0}^{s} p_{g}(\lambda)(\tau) \frac{(\theta(s)-\theta(\tau))^{k-1}}{(k-1) !} d \tau d s+b \varepsilon \int_{0}^{t} k(s) \frac{\theta(s)^{k-1}}{(k-1) !} d s \\
\leqq \frac{1}{k !} \int_{0}^{t} p_{g}(\lambda)(s) \int_{s}^{t} \frac{d}{d \tau}(\theta(\tau)-\theta(s))^{k} d \tau d s+\frac{b \varepsilon}{k !} \int_{0}^{t} \frac{d}{d s} \theta(s)^{k} d s \\
\leqq \frac{1}{k !} \int_{0}^{t} p_{g}(\lambda)(s)(\theta(t)-\theta(s))^{k} d s+\frac{b \varepsilon}{k !} \theta(t)^{k} .
\end{gathered}
$$

Summing up with respect to $k$, we get in the limit as $n \rightarrow \infty$,

$$
|x(\lambda)(t)-y(\lambda)(t)| \leqq b \varepsilon e^{\theta(t)}+\int_{0}^{t} p_{g}(\lambda)(s) \exp (\theta(t)-\theta(s)) d s, \quad t \in T .
$$

Q.E.D.

\section{Continuous relaxation theorem}

Using the parametric Filippov-Gronwall estimate established in section 4 (Theorem 4.1), we can now prove a continuous version of the relaxation theorem. In this section the parameter space $\Lambda$ is also separable (i.e. a Polish space).

Let $L_{w}^{1}(T, H)$ denote the space of equivalence classes of Bochner integrable functions $x: T \rightarrow H$ with the ("weak")-norm $\|x\|_{w}=\sup \left\{\left|\int_{t_{1}}^{t_{2}} x(s) d s\right|: 0 \leqq t_{1} \leqq t_{2} \leqq b\right\}$. The notation $\stackrel{\|\cdot\|_{w}}{\longrightarrow}$ stands for convergence in $L_{w}^{1}(T, H)$. Also if $h \in L^{q}(T, H)$, let $w(h) \in W_{p q}(T)$ be the unique solution of $\dot{x}(t)+A(t, x(t))=h(t)$ a.e., $x(0)=v_{0}$ for some given $v_{0} \in H$. We will need the following continuity result concerning $w(\cdot)$.

LEMmA. If hypothesis $H(A)$ holds, $\left\{h_{n}, h\right\}_{n \geqq 1} \subseteq L^{q}(T, H),\left|h_{n}(t)\right|,|h(t)| \leqq \psi(t)$ a.e. with $\phi \in L^{q}(T, \boldsymbol{R})$ and $h_{n} \stackrel{\|\cdot\|_{w}}{\longrightarrow} h$ as $n \rightarrow \infty$, then $w\left(h_{n}\right) \rightarrow w(h)$ in $C(T, H)$.

Proof. We know (see for example Lemma 3.1 of Aizicovici-Papageorgiou [1]), that $h_{n} \stackrel{w}{\rightarrow} h$ in $L^{q}(T, H)$. As before, because of the monotoncity of $A(t, \cdot)$, we get 


$$
\frac{1}{2}\left|w\left(h_{n}\right)(t)-w(h)(t)\right|^{2} \leqq \int_{0}^{t}\left(h_{n}(s)-h(s), w_{n}(h)(s)-w(h)(s)\right) d s .
$$

From the a priori bounds established in Papageorgiou [19] (see also Zeidler [23]), we know that $\left\{w\left(h_{n}\right)(\cdot)\right\}_{n \geq 1}$ is bounded in $W_{p q}(T)$, hence relatively compact in $L^{p}(T, H)$ (recall that since $X$ embeds compactly in $H, W_{p q}(T)$ embeds compactly in $L^{p}(T, H)$; see section 2$)$. Thus by passing to a subsequence if necessary, we may assume that $w\left(h_{n}\right) \rightarrow u$ in $L^{p}(T, H)$. Then we have

$$
\begin{gathered}
\frac{1}{2}\left|w\left(h_{n}\right)(t)-w(h)(t)\right|^{2} \leqq \int_{0}^{t}\left(h_{n}(s)-h(s), w\left(h_{n}\right)(s)-u(s)\right) d s \\
+\int_{0}^{t}\left(h_{n}(s)-h(s), u(s)-w(h)(s)\right) d s \rightarrow 0 \text { as } n \rightarrow \infty .
\end{gathered}
$$

By a standard contradiction argument we can show that $w\left(h_{n}\right) \rightarrow w(h)$ in $C(T, H)$.

Q.E. D.

So now we can state and prove our continuous relaxation theorem.

TheOREM 5.1. If hypotheses $H(A), H(F)_{2}, H_{0}$ hold, $\lambda \rightarrow[y(\lambda), g(\lambda)]$ is a continuous map from $A$ into $C(T, H) \times L^{q}(T, H)$ such that for all $\lambda \in \Lambda[y(\lambda), g(\lambda)] \in P_{r}(\lambda)$ and $\varepsilon>0$,

then there exists $\lambda \rightarrow x(\lambda)$ a continuous map from $\Lambda$ into $C(T, H)$ such that for all $\lambda \in \Lambda, \quad x(\lambda) \in S(\lambda)$ and $\|x(\lambda)-y(\lambda)\|_{C(T, H)}<\varepsilon$.

Proof. From the a priori estimates established in [19], we know that without any loss of generality, we may assume that $|F(t, x, \lambda)|=\sup \{|v|: v \in F(t, x, \lambda)\} \leqq$ $\leqq \phi(t)$ a.e. with $\phi \in L^{q}(T, R)$. Also from the lemma above we know that we can find $\delta>0$ such that if $h \in L^{q}(T, H)$ with $|h(t)| \leqq \phi(t)$ a.e. and $\|g(\lambda)-h\|_{w} \leqq \delta$, then we have $\|y(\lambda)-w(h, \lambda)\|_{C(T, H)} \leqq \varepsilon / 4 M \hat{b}$, where $M=e^{\theta(b)}$ and $\hat{b}=\max [b, 1]$.

Divide $T$ into subintervals $T_{k}=\left[t_{k}, t_{k+1}\right], k=0,1,2, \cdots, N$ such that $\int_{r_{k}} \phi(s) d s<\delta / 4$ with $\delta>0$ as above. The existence of such partition of $T$ is guaranteed by the absolute continuity of the Lebesque integral. Let $u(\lambda)(t)=$ $\int_{0}^{t} g(\lambda)(s) d s$. Then $u(\lambda)(\cdot) \in C(T, H)$ and by hypothesis $\lambda \rightarrow u(\lambda)\left(t_{k+1}\right)-u(\lambda)\left(t_{k}\right)$ is a continuous selector of the parametric set-valued integral $\int_{T_{k}} \overline{\operatorname{conv}} F(t, y(\lambda)(t), \lambda) d t$ which belongs in $P_{w k c}(H)$ (see the Corollary to Proposition 3.1 of Papageorgiou [12]). Recall (see for example Hiai-Umegaki [8], Corollary 4.3) that $\int_{T_{k}} \overline{\operatorname{conv}} F(t, y(\lambda)(t), \lambda) d t=c l \int_{T_{k}} F(t, y(\lambda)(t), \lambda) d t$. Then consider the multifunction

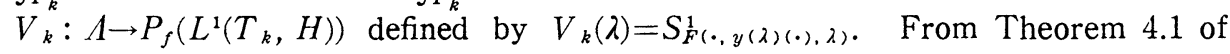
[14], we know that $\lambda \rightarrow V_{k}(\lambda)$ is l.s. $c$. and of course has decomposable values. So we can apply Theorem 1 of Fryszkowski-Rzezuchowski [7], to get $r_{k}: \Lambda \rightarrow$ 
$L^{1}\left(T_{k}, H\right)$ a continuous map such that $r_{k}(\lambda) \in V_{k}(\lambda)$ and $\mid \int_{T_{k}} g(\lambda)(t) d t-$ $\int_{T_{k}} r_{k}(\lambda)(t) d t \mid<\delta / 2 N, k=0,1,2, \cdots, N$. Set $f(\lambda)=\sum_{k=0}^{N} \chi_{T_{k}} r_{k}(\lambda) \in L^{q}(T, H)$ and $z(\lambda)$ $=w(f(\lambda), \lambda)$. Our claim is that $\|g(\lambda)-f(\lambda)\|_{w} \leqq \delta$. Indeed by definition we have

$$
\|g(\lambda)-f(\lambda)\|_{w}=\sup \left[\left|\int_{0}^{t}(g(\lambda)(s)-f(\lambda)(s)) d s\right|: 0 \leqq t \leqq b\right] .
$$

For $t \in T_{m}, m \in\{0,1,2, \cdots, N\}$, we have

$$
\begin{gathered}
\left|\int_{0}^{t}(g(\lambda)(s)-f(\lambda)(s)) d s\right| \\
\leqq\left|\int_{\substack { m-1 \\
\begin{subarray}{c}{m=T_{k} \\
k=0{ m - 1 \\
\begin{subarray} { c } { m = T _ { k } \\
k = 0 } }\end{subarray}}(g(\lambda)(s)-f(\lambda)(s)) d s\right|+\left|\int_{t_{m}}^{t}(g(\lambda)(s)-f(\lambda)(s)) d s\right| \\
<\frac{\delta}{2}+\int_{t_{m}}^{t} 2 \phi(s) d s<\frac{\delta}{2}+\frac{\delta}{2}=\delta \\
\Rightarrow\|g(\lambda)-f(\lambda)\|_{w} \leqq \delta \\
\Rightarrow\|y(\lambda)-z(\lambda)\|_{C(T, H)} \leqq \frac{\varepsilon}{4 M \hat{b}} .
\end{gathered}
$$

Observe the $f(\lambda)(t) \in F(t, y(\lambda)(t), \lambda)$ a.e. So using hypothesis $H(F)_{2}(2)$ we get $d(f(\lambda)(t), F(t, z(\lambda)(t), \lambda)) \leqq k(t)(\varepsilon / 4 M \hat{b})$. Then apply Theorem 4.1, with $p_{f}(\lambda)(\cdot)$ $=k(\cdot)(\varepsilon / 4 M \hat{b})$ to get a continuous map $\lambda \rightarrow x(\lambda)$ from $\Lambda$ into $C(T, H)$ such that $x(\lambda) \in S(\lambda)$ for all $\lambda \in A$ and

$$
\begin{gathered}
\|x(\lambda)-z(\lambda)\|_{C(T, H)} \leqq \\
\frac{\varepsilon}{4 M \hat{b}} M+\frac{\varepsilon}{4 M \hat{b}} M \int_{0}^{t} k(s) \exp (-\theta(s)) d s \\
\leqq \frac{\varepsilon}{4}+\frac{\varepsilon}{4}\left(1-e^{-\theta(t)}\right) \quad(\text { since } \hat{b} \geqq 1) \\
<\frac{\varepsilon}{4}+\frac{\varepsilon}{4}=\frac{\varepsilon}{2} .
\end{gathered}
$$

So finally we have

$$
\begin{gathered}
\|x(\lambda)-y(\lambda)\|_{C(T, H)} \leqq \\
\quad x(\lambda)-z(\lambda)\left\|_{C(T, H)}+\right\| z(\lambda)-y(\lambda) \|_{C(T, H)} \\
<\frac{\varepsilon}{2}+\frac{\varepsilon}{4 M \hat{b}}<\varepsilon .
\end{gathered}
$$

Q. E. D.

\section{Application}

As an application we consider a parametric controlled diffusion equation with nonlinear friction. We convexify its control constraint set (relaxed system) and examine whether we can approximate every relaxed trajectory by trajec- 
tories of the original system, uniformly in the time variable and continuously with respect to the parameter. So let $T=[0, b]$ and $Z$ a bounded domain in $\boldsymbol{R}^{N}$ with smooth boundary $\Gamma$. The system under consideration is the following:

$$
\left\{\begin{array}{c}
\frac{\partial x}{\partial t}-\Delta x-\sum_{\imath, j=1}^{N} D_{i}\left(r_{\imath j}\left(t,\|D x\|^{p-2}\right) D, x\right)=f(t, z, x(t, z), \lambda) u(t, z) \text { a.e. } \\
\left.x\right|_{T \times \Gamma}=0, \quad x(0, z)=x_{0}(z, \lambda) \\
u(t, \cdot) \in U(t, \lambda) \text { a.e., } p \geqq 2 .
\end{array}\right\}
$$

Here $D_{i}=\partial / \partial z_{\imath}, i=1,2, \cdots, N$ and $D x=\operatorname{grad} x=\left(D_{1} x, \cdots, D_{N} x\right)$. In connection with (4), we consider the same system, but with convexified control constraints; i. e.

$$
\left\{\begin{array}{c}
\frac{\partial x}{\partial t}-\Delta x-\sum_{\imath, j=1}^{N} D_{i}\left(r_{\imath j}\left(t,\|D x\|^{p-2}\right) D, x\right)=f(t, z, x(t, z), \lambda) u(t, z) \text { a.e. } \\
\left.x\right|_{T \times \Gamma}=0, \quad x(0, z)=x_{0}(z) \text { a.e. } \\
u(t, \cdot) \in \overline{\operatorname{conv}} U(t, \lambda) \text { a.e., } p \geqq 2 .
\end{array}\right\}
$$

We will need the following hypotheses on the data:

$H(r): \quad r: T \times \boldsymbol{R}_{+} \rightarrow \mathcal{L}\left(\boldsymbol{R}^{N}\right)$ is an $N \times N$-matrix valued map such that

(1) $t \rightarrow r(t, v)$ is measurable,

(2) $v \rightarrow r(t, v)$ is continuous,

(3) $\|r(t, v)\|_{\perp} \leqq a|v|$ with $a>0$,

(4) $\left(r\left(t,\|\xi\|^{p-2}\right) \xi-r\left(t,\left\|\xi^{\prime}\right\|^{p-2}\right) \xi^{\prime}, \xi-\xi^{\prime}\right)_{R^{N}} \geqq 0$ for all $\left(t, \xi, \xi^{\prime}\right) \in T \times \boldsymbol{R}^{N} \times \boldsymbol{R}^{N}$,

(5) $\hat{c}\|\xi\|^{p} \leqq\left(r\left(t,\|\xi\|^{p-2}\right) \xi, \xi\right)_{R^{N}}$ for all $(t, \xi) \in T \times \boldsymbol{R}^{N}$ and with $\hat{c}>0$.

$H(f): \quad f: T \times Z \times \boldsymbol{R} \times \Lambda \rightarrow \boldsymbol{R}$ is a function such that

(1) $(t, z) \rightarrow f(t, z, x, \lambda)$ is measurable,

(2) $\left|f(t, z, x, \lambda)-f\left(t, z, x^{\prime}, \lambda\right)\right| \leqq k(t, z)\left|x-x^{\prime}\right|$ a.e. with $k \in L^{1}(T \times Z, \boldsymbol{R})$,

(3) $|f(t, z, x, \lambda)| \leqq a_{1}(t, z)+c_{1}(z)|x|^{2 / q}$ a.e. with $a_{1} \in L^{q}(T \times Z, \boldsymbol{R}), c_{1} \in L^{\infty}(Z, \boldsymbol{R})$.

$H(U): \quad U: T \times \Lambda \rightarrow P_{w k}\left(L^{2}(Z, R)\right)$ is a multifunction such that

(1) $t \rightarrow U(t, \lambda)$ is measurable,

(2) $\lambda \rightarrow U(t, \lambda)$ is continuous,

(3) $|U(t, \lambda)|=\sup \left\{\|u\|_{2}: u \in U(t, \lambda)\right\} \leqq M, M>0$.

As in section 5 , the parameter space $\Lambda$ is a Polish space.

In this case, the evolution triple consists of $X=W_{0}^{1, p}(Z), H=L^{2}(Z)$ and $X^{*}=W^{-1, q}(Z)$. From the Sobolev embedding theorem we know that $X \leqq H \leqq X^{*}$ with all embeddings being continuous, dense and compact

Consider the following two Dirichlet forms:

$$
a_{1}: T \times W_{0}^{1, p}(Z) \times W_{0}^{1, p}(Z) \rightarrow \boldsymbol{R} \text { defined by }
$$




$$
\begin{aligned}
a_{1}(t, x, y) & =\int_{Z} \sum_{i, j=1}^{N} r_{i j}\left(t,\|D x\|^{p-2}\right) D_{\imath} x D_{j} y d z \\
& =\int_{Z}\left(r\left(t,\|D x\|^{p-2}\right) D x, D y\right)_{R^{N}} d z
\end{aligned}
$$

and $a_{2}: W_{0}^{1, p}(Z) \times W_{0}^{1, p}(Z) \rightarrow \boldsymbol{R}$ defined by

$$
a_{2}(x, y)=\int_{Z} \sum_{\imath=1}^{N} D_{\imath} x D_{\imath} y d z=\int_{Z}(D x, D y)_{R^{N}} d z .
$$

Note that because of hypothesis $H(r)$ (3), we have

$$
\left|a_{1}(t, x, y)\right| \leqq \int_{z} a\|D x\|^{p-1}\|D y\| d z .
$$

Using Hölder's inequality and recalling that $\|D x\|_{p}^{p}=\sum_{\imath=1}^{N}\left\|D_{\imath} x\right\|_{p}^{p}$ is an equivalent norm for $W_{0}^{1, p}(Z)$, we get that

$$
\left|a_{1}(t, x, y)\right| \leqq \hat{a}\|x\|_{W_{0}^{1, p}(Z)}^{p-1} \cdot\|y\|_{W_{0}^{1, p}(Z)} \quad \text { for some } \hat{a}>0 .
$$

So there exists $A_{1}: T \times W_{0}^{1, p}(Z) \rightarrow W^{-1, q}(Z)$ such that

$$
\left\langle A_{1}(t, x), y\right\rangle=a_{1}(t, x, y)
$$

and $\left\|A_{1}(t, x)\right\|_{W^{-1, q_{(Z)}}} \leqq \hat{a}\|x\|_{W_{0}^{1, p}(Z)}^{p-1}, \hat{a}>0$.

From Fubini's theorem, we know that $t \rightarrow a_{1}(t, x, y)$ is measurable $\Rightarrow t \rightarrow$ $\left\langle A_{1}(t, x), y\right\rangle$ is measurable for all $x, y \in W_{0}^{1, p}(Z) \Rightarrow t \rightarrow A_{1}(t, x)$ is weakly measurable from $T$ into $W^{-1, q}(Z)$. Since $W^{-1, q}(Z)$ is a separable reflexive Banach space (a separable Hilbert space if $p=q=2$ ), from the Pettis measurability theorem, we have that $t \rightarrow A_{1}(t, x)$ is measurable. Also if $x_{n} \rightarrow x$ in $W_{0}^{1, p}(Z) \Rightarrow$ $D x_{n} \rightarrow D x$ in $L^{p}\left(Z, \boldsymbol{R}^{N}\right) \Rightarrow r\left(t,\left\|D x_{n}(z)\right\|^{p-2}\right) \rightarrow r\left(t,\|D x(z)\|^{p-2}\right)$ in measure (cf. hypothesis $H(r)(2)$ ) and so by the dominated convergence theorem, we have that $\int_{Z} r\left(t,\left\|D x_{n}(z)\right\|^{p-2}\right) D x_{n} D y d z \rightarrow \int_{z} r\left(t,\|D x(z)\|^{p-2}\right) D x D y d z \Rightarrow\left\langle A_{1}\left(t, x_{n}\right), y\right\rangle-$, $\left\langle A_{1}(t, x), y\right\rangle \Rightarrow A_{1}(t, \cdot)$ is demicontinuous, hence hemi-continuous (see Zeidler [23], p. 596). Also note that because of hypothesis $H(r)(4)$, we have

$$
\begin{gathered}
\left\langle A_{1}(t, x)-A_{1}(t, y), x-y\right\rangle \\
=\int_{Z}\left(r\left(t,\|D x\|^{p-2}\right) D x-r\left(t,\|D y\|^{p-2}\right) D y, D x-D y\right)_{R^{N}} d z \geqq 0
\end{gathered}
$$

$\Rightarrow x \rightarrow A_{1}(t, x)$ is monotone.

Finally from hypothesis $H(r)(5)$, we get

$$
\hat{c}_{1}\|x\|^{p} \leqq\left\langle A_{1}(t, x), x\right\rangle \quad \text { with } \hat{c}_{1}>0 .
$$

Next for Dirichlet form $a_{2}(x, y)$, via the Cauchy-Schwartz inequality and since $L^{p}(Z, \boldsymbol{R}) \leqq L^{2}(Z, \boldsymbol{R})$ with continuous injection (because $2 \leqq p<\infty$ ), we get 


$$
\left|a_{2}(x, y)\right| \leqq \eta\|x\|_{W_{0}^{1, p}(Z)}\|y\|_{W_{0}^{1, p}(Z)} \quad \text { for some } \eta>0 \text {. }
$$

So there exists $A_{2} \in \mathcal{L}\left(X, X^{*}\right)$ such that

$$
a_{2}(x, y)=\left\langle A_{2} x, y\right\rangle
$$

and from Poincare's inequality, we have that

$$
\hat{c}_{2}\|x\|_{W_{0}^{1, p}(Z)}^{2} \leqq\left\langle A_{2}, x, x\right\rangle .
$$

Thus if we set $A(t, x)=A_{1}(t, x)+A_{2}(t, x)$, we see that $A(t, x)$ satisfies hypothesis $H(A)$.

Next let $\hat{f}: T \times H \times \Lambda \rightarrow H$ be defined by

$$
\hat{f}(t, x, \lambda)(\cdot)=f(t, \cdot, x(\cdot), \lambda)
$$

i. e. $\hat{f}(t, x, \lambda)$ is the Nemitsky (superposition) operator corresponding to $f$.

Let $F: T \times H \times \Lambda \rightarrow P_{w k}(H)$ be defined by

$$
F(t, x, \lambda)=\hat{f}(t, x, \lambda) U(t, \lambda)=\{\hat{f}(t, x, \lambda) u ; u \in U(t, \lambda)\} .
$$

Using hypotheses $H(f)$ and $H(U)$, we can easily check that $F(t, x, \lambda)$ satisfies hypothesis $H(F)_{2}$. A straight forward application of Aumann's selection theorem shows that (4), is equivalent to the following abstract evolution inclusion (i.e. control free problem):

$$
\left\{\begin{array}{c}
\dot{x}(t)+A(t, x(t)) \in F(t, x(t), \lambda) \text { a.e. } \\
x(0)=\hat{x}_{0}(\lambda)
\end{array}\right\}
$$

with $\hat{x}_{0}(\lambda)(\cdot)=x_{0}(\cdot, \lambda) \in L^{2}(Z)=H$. Similarly (5) is equivalent to

$$
\left\{\begin{array}{c}
\dot{x}(t)+A(t, x(t)) \in \overline{\operatorname{conv}} F(t, x(t), \lambda) \text { a.e. } \\
x(0)=\hat{x}_{0}(\lambda)
\end{array}\right\}
$$

the convexified version of $(4)^{\prime}$.

We will make a final hypothesis concerning $x_{0}(\cdot, \lambda)$.

$H_{0}^{\prime}: \lambda \rightarrow x_{0}(\cdot, \lambda)=\hat{x}_{0}(\lambda)$ is continuous from $\Lambda$ into $H=L^{2}(Z)$.

Applying Theorem 5.1 on the pair of systems (4) $)^{\prime}$ and $(5)^{\prime}$ which is equivalent to the pair (4) and (5), we get the following parametric approximation result for the latter :

THEOREM 6.1. If hypotheses $H(r), H(f), H(U)$ and $H_{0}^{\prime}$ hold, $\lambda \rightarrow[x(\lambda), u(\lambda)]$ is a continuous map from $\Lambda$ into $C\left(T, L^{2}(Z)\right) \times L^{2}(T \times Z, R)$ such that for every $\lambda \in \Lambda$, the pair $[x(\lambda), u(\lambda)]$ satisfies (5) (i.e. is an admissible "state-control" pair for (5)) and $\varepsilon>0$, 
then we can find $\lambda \rightarrow y(\lambda)$ a continuous map from $\Lambda$ into $C\left(T, L^{2}(Z)\right)$ so that for every $\lambda \in \Lambda, y(\lambda)(\cdot)$ is a state trajectory of (4) and

$$
\sup _{t \in T} \int_{Z}|x(\lambda)(t, z)-y(\lambda)(t, z)|^{2} d z<\varepsilon
$$

Acknowledgement. The author wishes to express his gratitude to the referee for his/her many corrections and constructive criticism.

\section{REFERENCES}

[1] S. Aizicovici And N.S. Papageorgiou, Extremal solutions to a class of multivalued integral equations in a Banach space, J. Appl. Math. Stochastic Anal., 5 (1992), 205-220.

[2] J.-P. Aubin ANd A. Cellina, Differential Inclusions, Springer-Verlag, Berlin, 1984.

[3] A. Bressan And G. Colombo, Extensions and selections of maps with decomposable values, Studia Math., 90 (1988), 69-86.

[4] H. Brezis, Operateurs Maximaux Monotones, North Holland, Amsterdam, 1973.

[5] F.S. DEBlasi AND J. MYJAK, On continuous approximations for multifunctions, Pacific J. Math., 123 (1986), 9-31.

[6] H. Frankowska, A priori estimates for operational differential inclusions, J. Differential Equations, 84 (1990), 100-128.

[7] A. Fryszkowski and T. Rzezuchowski, Continuous version of Filippov-Wazewski relaxation theorem, J. Differential Equations, 94 (1991), 254-265.

[8] F. HIAI AND H. UMEGAKI, Integrals, conditional expectations and martingales of multivalued mappings, J. Multivariate Anal., 7 (1977), 149-182.

[9] S. Hu, V. Lakshmikantham and N.S. Papageorgiou, On the solution set of nonlinear evolution inclusions, Dynamic Systems Appl., 1 (1992), 71-82.

[10] E. KLein And A. Thompson, Theory of Correspondences, Wiley, New York, 1984.

[11] N.S. Papageorgiou, Optimal control of nonlinear evolution inclusions, J. Optim. Theory Appl., 67 (1990), 321-354.

[12] N.S. PAPAgeorgiou, On the theory of Banach space-valued multifunctions, Part 1: Integration and conditional expectation, J. Multivariate Appl., 17 (1985), 185-206.

[13] N.S. PAPAgEorgiou, On measurable multifunctions with applications to random multivalued equations, Math. Japon., 32 (1987), 437-464.

[14] N.S. PAPAgEorgiou, Convergence theorems for Banach space-valued integrable multifunctions, Internat. J. Math. Math. Sci., 10 (1985), 433-442.

[15] N.S. PAPAgEorgiou, A relaxation theorem for differential inclusions in Banach spaces, Tôhoku Math. J., 39 (1987), 505-517.

[16] N.S. PAPAgeorgiou, Relaxation of infinite dimensional variational and control problems with state constrains, Koda1 Math. J., 12 (1989), 392-419.

[17] N.S. PAPAgEorgiou, Volterra integrodifferential inclusions in reflexive Banach spaces, Funkcial. Ekvac., 34 (1991), 257-277.

[18] N.S. Papageorgiou, Continuous dependence results for a class of evolution inclusions, Proc. Edinburgh Math. Soc., 35 (1992), 139-158. 
[19] N.S. PAPAgeorgiou, On the bang-bang principle for nonlinear evolution inclusions, Aequationes Math., 45 (1993), 267-280.

[20] N.S. Papageorgiou, Convexity of the orientor field and the solution set of a class of evolution inclusions, to appear in Math. Slovaca.

[21] J. Simon, Compact sets in the space $L^{p}(T, B)$, Ann. Mat. Pura Appl., 125 (1986), 65-96.

[22] D. WAGNER, Survey of measurable selection theorems, SIAM J. Control. Optim., 15 (1977), 859-903.

[23] E. Zeidler, Nonlinear Functional Analysıs and its Applications II, Springer, New York, 1990.

[24] Q.-J. $Z_{H U}$, On the solution set of differential inclusions in Banach spaces, J. Differential Equations, 93 (1991), 213-237.

[25] G. Choquet, Lectures on Analysis, Vol. 1, W. Benjamin, Inc., London, 1969.

\author{
(Permanent Address) \\ National Technical University \\ Department of Mathematics \\ ZOGRAFOU CAMPUS \\ Athens 15780, Greece \\ and \\ Florida institute of Tecinology \\ Department of Applied Mathematics \\ 150 West University Blvd. \\ Melbourne, Florida 32901-6988, U.S.A.
}

